\title{
The Myerson Value for Complete Coalition Structures*
}

\author{
Takashi $\mathrm{Ui}^{\dagger} \quad$ Hiroyuki Kojima ${ }^{\ddagger} \quad$ Atsushi Kajii ${ }^{\S}$
}

June 2011

\begin{abstract}
In order to describe partial cooperation structures, this paper introduces complete coalition structures as sets of feasible coalitions. A complete coalition structure has a property that, for any coalition, if each pair of players in the coalition belongs to some feasible coalition contained in the coalition then the coalition itself is also feasible. The union stable structures, which constitute the domain of the Myerson value, are a special class of the complete coalition structures. As an allocation rule on complete coalition structures, this paper proposes an extension of the Myerson value for complete coalition structures and provides an axiomatization.
\end{abstract}

JEL classification: C71.

Keywords: union stable structure; complete coalition structure; the Myerson value; potential.

*An earlier version of this paper was circulated under the title "A Refinement of the Myerson Value." We are very grateful to the referee for many valuable comments. We also thank Yuji Fujinaka and Yukihiko Funaki for valuable comments and discussions. Ui acknowledges Grant-in-Aid for Scientific Research (C) (No. 20530150). Kojima acknowledges Grant-in-Aid for Scientific Research (C) (No. 22530186). Kajii acknowledges Grant-in-Aid for Scientific Research (S) (No. 90152298). This work was done partially while Kajii and Ui were visiting the Institute for Mathematical Sciences, National University of Singapore in 2005. The visit was supported by the Institute.

${ }^{\dagger}$ Faculty of Economics, Yokohama National University, 79-3 Tokiwadai, Hodogaya-ku, Yokohama 240-8501, Japan. E-mail: oui@ynu.ac.jp (T. Ui). Phone: +81-45-339-3531. Fax: +81-45-339-3574.

${ }^{\ddagger}$ Department of Economics, Teikyo University. E-mail: hkojima@main.teikyo-u.ac.jp (H. Kojima).

$\S$ Institute of Economic Research, Kyoto University. E-mail: kajii@kier.kyoto-u.ac.jp (A. Kajii). 


\section{Introduction}

One way to describe the structure of partial cooperation in the context of cooperative games is to specify sets of feasible coalitions with some appropriate properties. Algaba et al. (2000) considered union stable structures as such sets. A union stable structure has the property that the union of two intersecting feasible coalitions is also feasible, which can be interpreted as follows: players who are common members of two feasible coalitions are able to act as intermediaries to elicit cooperation among all the players in either of these coalitions, and so their union should be a feasible unit of cooperation as well. As shown by Algaba et al. (2000), for a given set of feasible coalitions, there exists a minimal union stable structure including the set. It consists of feasible coalitions evoked by intermediaries as well as the original ones. Furthermore, such a minimal union stable structure is unique. This implies that sets of feasible coalitions with the same minimal union stable structure containing them form an equivalence class.

The union stable structures are essentially the domain of the Myerson value, as shown by Algaba et al. (2001). The Myerson value introduced by Myerson (1977) is a solution for cooperative games under the partial cooperation structures described by networks, i.e., sets of two-player coalitions. Myerson (1980) and van den Nouweland et al. (1992) considered the partial cooperation structures described by sets of coalitions (not necessarily two-player coalitions), and studied the Myerson value for them. The Myerson value in the sense of Myerson (1980) and van den Nouweland et al. (1992) assigns the same payoff vectors to all sets of coalitions belonging to the same equivalence class represented by a minimal union stable structure, which implies that the union stable structures are essentially the domain of the Myerson value. On the basis of this observation, Algaba et al. (2001) introduced the Myerson value for union stable structures and provided an axiomatization for it.

In this paper, we introduce complete coalition structures as sets of feasible coalitions. A complete coalition structure has a property with the following interpretation: given a set of feasible coalitions, if each pair of players in a group of players can work together in some feasible coalition contained in the group, then the group itself becomes a feasible unit of cooperation. It can be shown that any union stable structure is a complete coalition structure, but not every complete coalition structure is a union stable structure. Thus, the complete coalition structures are a strictly larger class than the union stable structures.

The study of complete coalition structures is motivated by our observation that the requirement of union stable structures is too strong in some cases. Coming back to the above interpretation of union stable structures, one may wish to describe a situation where the cost for the intermediaries is too high to elicit cooperation because the two feasible coalitions contain a great number of players with only a few common players. Also, it is interesting to consider a situation where only limited communication channels are available because the distance or direction of communication is restricted. For example, if players are located along a very long line and only adjacent players can communicate, or if players are partially ordered (as in a hierarchical organization) and only comparable players can communicate, then all the players may not be able to share the information necessary for cooperation. Complete coalition structures can differentiate these situations, while union stable structures cannot.

This paper proposes an extension of the Myerson value for complete coalition structures and provides an axiomatization for it. The Myerson value for complete coalition structures coincides with the Myerson value over the union stable structures, but it also assigns payoff vectors to those complete coalition structures that are not union stable structures. Thus, it provides a method of 
more refined assignments of payoff vectors than the Myerson value for union stable structures.

The organization of the paper is as follows. Preliminary definitions and results are summarized in Section 2. Complete coalition structures are introduced in Section 3. The main result is stated in Section 4 and is proved in Section 5. Section 6 concludes.

\section{Preliminaries}

Let $N=\{1, \ldots, n\}$ be a set of players. A game $v$ is a function from $2^{N}$ to $\mathbb{R}$ with $v(\emptyset)=0$. A nonempty subset $S \in 2^{N}$ is a coalition, and by convention, $2^{N}$ is interpreted as the set of all coalitions. The unanimity game on a coalition $T$ is denoted by $u_{T}$ and defined as

$$
u_{T}(S)= \begin{cases}1 & \text { if } T \subseteq S \\ 0 & \text { otherwise }\end{cases}
$$

Each game $v$ is uniquely represented as a linear combination of unanimity games (Shapley, 1953):

$$
v=\sum_{T \in 2^{N}} \beta_{T} u_{T} \text { where } \beta_{T}=\sum_{S \subseteq T}(-1)^{|T|-|S|} v(S) .
$$

The coefficient $\beta_{T}$ in (1) is called the dividend of a coalition $T$ for the game $v$.

Let $\mathcal{H} \subseteq 2^{N}$ be a set of coalitions. We write $\mathcal{H}_{S}=\{H \in \mathcal{H} \mid H \subseteq S\}$ and $\mathcal{H}_{-i}=\mathcal{H}_{N \backslash\{i\}}$ for $S \in 2^{N}$ and $i \in N$, respectively. For a game $v$ and a set of coalitions $\mathcal{H}$, we consider another game $v^{\mathcal{H}}$ as defined below.

Definition 1 A game $v^{\mathcal{H}}=\sum_{T \in 2^{N}} \beta_{T}^{\mathcal{H}} u_{T}$ is the $\mathcal{H}$-projected game of $v$ if $\beta_{T}^{\mathcal{H}}$ is determined recursively by the following rule:

1. $\beta_{\{i\}}^{\mathcal{H}}=v(\{i\})$ if $\{i\} \in \mathcal{H}$ and $\beta_{\{i\}}^{\mathcal{H}}=0$ otherwise.

2. For $T \in 2^{N}$ with $|T| \geq 2, \beta_{T}^{\mathcal{H}}=v(T)-\sum_{S \subsetneq T} \beta_{S}^{\mathcal{H}}$ if $T \in \mathcal{H}$ and $\beta_{T}^{\mathcal{H}}=0$ otherwise.

Note that the above rule is rewritten as $v^{\mathcal{H}}(S)=v(S)$ for each $S \in \mathcal{H}$ and $\beta_{T}^{\mathcal{H}}=0$ for each $T \notin \mathcal{H}$. This implies the following lemma.

Lemma 1 The $\mathcal{H}$-projected game $v^{\mathcal{H}}=\sum_{T \in 2^{N}} \beta_{T}^{\mathcal{H}} u_{T}$ is a unique game satisfying the following conditions: $v^{\mathcal{H}}(S)=v(S)$ for each $S \in \mathcal{H}$ and $\beta_{T}^{\mathcal{H}}=0$ for each $T \notin \mathcal{H}$.

This lemma states that the $\mathcal{H}$-projected game of $v$ is in fact a projection in the following sense. Let the space of all games be parametrized in such a way that the set of parameters of $v=\sum_{T \in 2^{N}} \beta_{T} u_{T}$ is $\{v(S)\}_{S \in \mathcal{H}} \cup\left\{\beta_{T}\right\}_{T \notin \mathcal{H}}$. The game $v^{\mathcal{H}}$ is the orthogonal projection of $v$ to the subspace of games with $\beta_{T}=0$ for each $T \notin \mathcal{H}$.

The Shapley value of a game $v$ is the payoff vector $\phi(v) \in \mathbb{R}^{N}$ given by the following formula (Shapley, 1953):

$$
\phi_{i}(v)=\sum_{S \in 2^{N}: i \in S} \frac{(|S|-1) !(|N|-|S|) !}{|N| !}(v(S)-v(S \backslash\{i\})) \text { for each } i \in N .
$$

In particular, the Shapley value of $u_{T}$ is given by

$$
\phi_{i}\left(u_{T}\right)= \begin{cases}1 /|T| & \text { if } i \in T, \\ 0 & \text { otherwise. }\end{cases}
$$


Since the Shapley value is linear in games, we have an alternative formula for the Shapley value of $v=\sum_{T \in 2^{N}} \beta_{T} u_{T}$ which is as follows:

$$
\phi_{i}(v)=\sum_{T \in 2^{N}} \beta_{T} \phi_{i}\left(u_{T}\right)=\sum_{T \in 2^{N}: i \in T} \beta_{T} /|T| .
$$

For a game $v$, consider another game $p$ satisfying

$$
\sum_{i \in S}(p(S)-p(S \backslash\{i\}))=v(S)
$$

for each $S \in 2^{N}$. Hart and Mas-Colell (1989) showed that $p$ exists uniquely and called it the potential for $v .^{1}$

Proposition 1 For a game $v=\sum_{T \in 2^{N}} \beta_{T} u_{T}$, there exists a unique game $p$ satisfying (3), which is given by

$$
p=\sum_{T \in 2^{N}} \frac{\beta_{T}}{|T|} u_{T}
$$

Furthermore, the vector of the marginal contributions $(p(N)-p(N \backslash\{i\}))_{i \in N}$ coincides with the Shapley value of $v$; that is,

$$
\phi_{i}(v)=p(N)-p(N \backslash\{i\}) \text { for each } i \in N .
$$

\section{Complete coalition structures}

We regard $\mathcal{H} \subseteq 2^{N}$ as a set of feasible coalitions that describes a partial cooperation structure. For example, consider $\{\{1,2\},\{2,3\},\{3,1\}\}$, where each pair of players in $\{1,2,3\}$ can work together in terms of some feasible coalition contained in $\{1,2,3\}$. In our concept of feasible coalitions defined below, the coalition $\{1,2,3\}$ is deemed to be also feasible, and $\{\{1,2\},\{2,3\},\{3,1\},\{1,2,3\}\}$ is the set of all feasible coalitions. We call such a set a complete coalition structure.

To provide a formal definition of a complete coalition structure, we introduce a concept of " $\mathcal{H}$ associated" relation in a coalition. Given a set of feasible coalitions $\mathcal{H}$, we say that a pair of players in a coalition $S$ are $\mathcal{H}$-associated in $S$ if there exists a feasible coalition in $\mathcal{H}_{S}$ containing the pair, in terms of which they can communicate and cooperate with each other and work together. ${ }^{2}$

Definition 2 For $\mathcal{H} \subseteq 2^{N}$ and $S \in 2^{N}, i, j \in S$ are $\mathcal{H}$-associated in $S$ provided there exists $H \in \mathcal{H}_{S}$ with $i, j \in H$.

We say that a coalition $S$ is $\mathcal{H}$-complete if any pair of players in $S$ are $\mathcal{H}$-associated in $S$; that is, the " $\mathcal{H}$-associated" relation in $S$ is complete.

Definition 3 For $\mathcal{H} \subseteq 2^{N}, S \in 2^{N}$ is $\mathcal{H}$-complete provided any $i, j \in S$ are $\mathcal{H}$-associated in $S$.

Given a set of feasible coalitions $\mathcal{H}$, a $\mathcal{H}$-complete coalition is deemed to be also feasible. We define a complete coalition structure as a set of all feasible coalitions in this sense.

\footnotetext{
${ }^{1}$ Originally, Hart and Mas-Colell (1989) defined a potential as a real-valued function on the space of games. The value assigned by the potential to the restriction of a game $v$ to a coalition $S$ corresponds to $p(S)$ in this paper.

${ }^{2}$ In the literature, $i, j \in S$ are said to be $\mathcal{H}$-connected in $S$ if there exist $H_{1}, \ldots, H_{m} \in \mathcal{H}_{S}$ such that $i \in H_{1}$, $j \in H_{m}$, and $H_{k} \cap H_{k+1} \neq \emptyset$ for $k=1, \ldots, m-1$. Note that if $i, j \in S$ are $\mathcal{H}$-associated in $S$ then they are $\mathcal{H}$-connected in $S$, but not vice versa.
} 
Definition 4 A set of coalitions $\mathcal{H} \subseteq 2^{N}$ is a complete coalition structure provided it is the set of all $\mathcal{H}$-complete coalitions.

It is straightforward to see that $\{\{1,2\},\{2,3\},\{3,1\},\{1,2,3\}\}$ is a complete coalition structure, whereas $\{\{1,2\},\{2,3\},\{3,1\}\}$ is not.

The set of all $\mathcal{H}$-complete coalitions contains $\mathcal{H}$ because each $S \in \mathcal{H}$ is a $\mathcal{H}$-complete coalition. As the next lemma shows, the set of all $\mathcal{H}$-complete coalitions is the minimal complete coalition structure containing $\mathcal{H}$. For a proof, see Kajii et al. (2007) who introduced the concept of $\mathcal{H}$ completeness. $^{3}$

Lemma 2 For $\mathcal{H} \subseteq 2^{N}$, the set of all $\mathcal{H}$-complete coalitions is a complete coalition structure.

Let CCS denote the set of all complete coalition structures. The following property of CCS is important in this paper.

Lemma 3 If $\mathcal{H} \in \mathbf{C C S}$, then $\mathcal{H}_{S} \in \mathbf{C C S}$ for each $S \in 2^{N}$.

Proof. It is enough to show that $H \in \mathcal{H}_{S}$ if and only if $H$ is $\mathcal{H}_{S}$-complete. Each $H \in \mathcal{H}_{S}$ is $\mathcal{H}_{S}$-complete by definition. Let $H \in 2^{N}$ be $\mathcal{H}_{S}$-complete, which implies that $H \subseteq S$. Since every $\mathcal{H}_{S}$-complete coalition is $\mathcal{H}$-complete, we must have $H \in \mathcal{H}$. Thus, $H \in \mathcal{H}_{S}$.

There are some other feasible coalition structures which are complete coalition structures, including union stable structures introduced by Algaba et al. (2000).

Definition 5 A set of coalitions $\mathcal{H} \subseteq 2^{N}$ is a union stable structure provided $S, T \in \mathcal{H}$ with $S \cap T \neq \emptyset$ implies $S \cup T \in \mathcal{H}$.

Let USS denote the set of all union stable structures. It is straightforward to check that if $\mathcal{H} \in$ USS, then $\mathcal{H}_{S} \in$ USS for each $S \in 2^{N}$. The next lemma shows that every union stable structure is a complete coalition structure.

Lemma 4 The set of all union stable structures USS is contained in CCS.

Proof. Let $\mathcal{H} \in$ USS. We show that $\mathcal{H} \in$ CCS. Since each $S \in \mathcal{H}$ is $\mathcal{H}$-complete by definition, it is enough to show that if $S \in 2^{N}$ is $\mathcal{H}$-complete then $S \in \mathcal{H}$. Without loss of generality, let $S=\{1, \ldots, k\}$. If $k=1$ and any $i, j \in S$ are $\mathcal{H}$-associated in $S$, then there exists $H \in \mathcal{H}_{S}$ with $1 \in H$, which implies that $S=H \in \mathcal{H}$. Suppose that $k \geq 2$. For each $i \in S \backslash\{k\}$, there exists $H_{i} \in \mathcal{H}_{S}$ with $i, i+1 \in H_{i}$. Since $H_{i} \cap H_{i+1} \neq \emptyset$ and $\mathcal{H}$ is a union stable structure, $S=\bigcup_{i=1}^{k-1} H_{i} \in \mathcal{H}$.

To illustrate the difference between complete coalition structures and union stable structures, let $S_{1}, S_{2} \subsetneq N$ be such that $\left|S_{1}\right| \geq 2,\left|S_{2}\right| \geq 2, S_{1} \cup S_{2} \subsetneq N$, and $S_{1} \cap S_{2}=\{n\}$. Consider $\mathcal{H}^{1}=\left\{S_{1}, S_{2}, N\right\}$ and $\mathcal{H}^{2}=\left\{S_{1}, S_{2}, S_{1} \cup S_{2}, N\right\}$. Then, we have $\mathcal{H}^{1}, \mathcal{H}^{2} \in \mathbf{C C S}, \mathcal{H}^{1} \notin \mathbf{U S S}$, and $\mathcal{H}^{2} \in$ USS. Note that $S_{1} \cup S_{2}$ is feasible in $\mathcal{H}^{2}$, but not in $\mathcal{H}^{1}$. To consider in what situations $S_{1} \cup S_{2}$ is not feasible, let $\mathcal{H}^{1}$ be an initially given set of feasible coalitions. If player $n$, who is a sole player in $S_{1} \cap S_{2}$, can act as an intermediary, then $S_{1} \cup S_{2}$ is also feasible, and thus the resulting set of feasible coalitions is $\mathcal{H}^{2}$. However, if player $n$ cannot do so, then the resulting set

\footnotetext{
${ }^{3}$ Kajii et al. (2007) use $\mathcal{H}$-completeness in their study of the Choquet integral. Another application of $\mathcal{H}$ completeness is found in Kajii et al. (2008).
} 
of feasible coalitions is $\mathcal{H}^{1}$. This might be the case when $\left|S_{1}\right|$ and $\left|S_{2}\right|$ are very large and the cost for player $n$ to act as an intermediary is very high.

Note that if $\mathcal{H}$ is closed in a union operation (i.e. $S \cup T \in \mathcal{H}$ for any $S, T \in \mathcal{H}$ ) then $\mathcal{H}$ is a union stable structure and thus it is a complete coalition structure. For example, antimatroids (Algaba et al., 2004) and the feasible coalition structures derived from permission structures (Gilles and Owen, 1999; Gilles et al., 1992) or precedence constraints (Faigle and Kern, 1992) are closed in a union operation. Therefore, these are all complete coalition structures. ${ }^{4}$

Other examples of complete coalition structures can be derived from a preorder $\preceq$ on $\mathrm{N}^{5}$ which are different from the feasible coalition structures derived from permission structures or precedence constraints. ${ }^{6}$ The next two general lemmas show that a set of intervals is a complete coalition structure even if it is not closed in a union operation.

Lemma 5 Let $\preceq$ be a preorder on $N$. If $\mathcal{H} \subseteq\{\{k: k \preceq i\}: i \in N\}$, then $\mathcal{H} \in \mathbf{C C S}$.

Proof. We write $i \sim j$ if $i \preceq j$ and $j \preceq i$, and $i \prec j$ if $i \preceq j$ but not $j \preceq i$. The binary relation $\prec$ is irreflexive by construction, and transitive since $\preceq$ is transitive. Note that any $S \in 2^{N}$ has $\bar{i} \in S$ such that there is no $j \in S$ with $\bar{i} \prec j$ since $S$ is a finite set. We call such $\bar{i}$ a maximal element of $S$ following the corresponding concept on a partially ordered set.

Let $S$ be a $\mathcal{H}$-complete coalition with a maximal element $\bar{i}$. By $\mathcal{H}$-completeness, for any $j \in S$, there exists $\bar{m} \in S$ such that $\bar{i}, j \in\{k: k \preceq \bar{m}\} \in \mathcal{H}_{S}$. By the property of $\bar{i}, \bar{m} \sim \bar{i}$ must follow. Hence, $\{k: k \preceq \bar{i}\}=\{k: k \preceq \bar{m}\} \in \mathcal{H}_{S}$ contains each $j \in S$. Since $\{k: k \preceq \bar{i}\} \subseteq S$, we have $S=\{k: k \preceq \bar{i}\} \in \mathcal{H}$.

For example, let $\preceq$ be such that $1 \preceq 2,2 \preceq 4,1 \preceq 3$, and $3 \preceq 4$. By the above lemma, $\mathcal{H}^{1}=$ $\{\{1,2\},\{1,3\},\{1,2,3,4\}\}$ is a complete coalition structure, which is not a union stable structure. The minimal union stable structure containing $\mathcal{H}^{1}$ is $\mathcal{H}^{2}=\{\{1,2\},\{1,3\},\{1,2,3\},\{1,2,3,4\}\}$. The difference is that $\{1,2,3\}$ is feasible in $\mathcal{H}^{2}$, but not in $\mathcal{H}^{1}$. To interpret this, imagine that $\preceq$ describes the possible direction of information flow between players: players $i$ and $j$ can share the same information if $i \preceq j$. Assume that a coalition is feasible if every player in the coalition can share the same information; that is, it is of the form $\{k: k \preceq i\}$. Then, $\{1,2,3\}$ is not of this form and thus is not feasible.

Lemma 6 Let $\preceq$ be a preorder on $N$. If $\mathcal{H} \subseteq\{\{k: i \preceq k \preceq j\}: i, j \in N\}$, then $\mathcal{H} \in \mathbf{C C S}$.

Proof. Let $\sim$ and $\prec$ be the binary relations as in the proof of Lemma 5 . Let $S$ be a $\mathcal{H}$-complete coalition with a maximal element $\bar{i}$ and a minimal element $\underline{i}$. By $\mathcal{H}$-completeness, for any $j \in S$, there exist $\underline{m}, m \in S$ such that $\underline{i}, j \in\{k: \underline{m} \preceq k \preceq m\} \in \mathcal{H}_{S}$, and there exist $\bar{m}, m \in S$ such that $\bar{i}, j \in\{k: m \preceq k \preceq \bar{m}\} \in \mathcal{H}_{S}$. By the property of $\underline{i}$ and $\bar{i}, \underline{m} \sim \underline{i}$ and $\bar{m} \sim \bar{i}$ must follow. This implies that $\underline{i} \preceq j \preceq \bar{i}$ for all $j \in S$. On the other hand, there exist $\underline{m}^{\prime}, \bar{m}^{\prime} \in S$ such that $\underline{i}, \bar{i} \in\left\{k: \underline{m}^{\prime} \preceq k \preceq \bar{m}^{\prime}\right\} \in \mathcal{H}_{S}$ by $\mathcal{H}$-completeness. Since $\underline{m}^{\prime} \sim \underline{i}, \bar{m}^{\prime} \sim \bar{i}$, and $\{k: \underline{i} \preceq k \preceq \bar{i}\}=\left\{k: \underline{m}^{\prime} \preceq k \preceq \bar{m}^{\prime}\right\} \subseteq S$, we must have $S=\{k: \underline{i} \preceq k \preceq \bar{i}\} \in \mathcal{H}$.

For example, let $\preceq$ be such that $i \preceq j$ if $i \leq j$. For $n \geq 3$, consider $\mathcal{H}^{1}=\{N\} \cup\{\{k, k+1\}\}_{k=1}^{n-1}$, $\mathcal{H}^{2}=\mathcal{H}^{1} \cup\{\{k, k+1, k+2\}\}_{k=1}^{n-2}$, and $\mathcal{H}^{m}=\mathcal{H}^{m-1} \cup\{\{k, \ldots, k+m\}\}_{k=1}^{n-m}$ for $2 \leq m \leq n-2$. By the above lemma, $\mathcal{H}^{m} \in \mathbf{C C S}$ for $1 \leq m \leq n-2$. On the other hand, $\mathcal{H}^{m} \notin \mathbf{U S S}$ for $m \neq n-2$

\footnotetext{
${ }^{4}$ We thank the referee for suggesting this discussion.

${ }^{5} \mathrm{~A}$ binary relation is a preorder if it is reflexive and transitive.

${ }^{6}$ Both a permission structure and a precedence constraint are interpreted as a preorder.
} 
and $\mathcal{H}^{n-2} \in$ USS. In $\mathcal{H}^{n-2}$, any coalition of the form $\{k, \ldots, k+l\}$ is feasible. In $\mathcal{H}^{m}$ with $m \neq n-2$, a coalition of the form $\{k, \ldots, k+l\}$ is feasible if $l \leq m$. This can be interpreted as follows. Let $\mathcal{H}^{1}$ be an initially given set of feasible coalitions and imagine that $n$ is very large. In $\mathcal{H}^{n-2},\{1, \ldots, n-1\}$ is feasible, where player 1 and player $n-1$ can communicate with each other through $n-2$ intermediaries, who must pass on information accurately. On the other hand, in $\mathcal{H}^{m}$, it is enough for $m-1$ intermediaries to pass on information accurately. Therefore, in some cases with very large $n$, it is natural to assume that $\mathcal{H}^{m}$ is the set of feasible coalitions for some $m \neq n-2$, rather than $\mathcal{H}^{n-2}$.

\section{The Myerson value for complete coalition structures}

Given a game $v$, we consider allocation rules on complete coalition structures. An allocation rule on complete coalition structures is a mapping $f: \mathbf{C C S} \rightarrow \mathbb{R}^{N}$ such that if $i \notin S$ for each $S \in \mathcal{H}$ then $f_{i}(\mathcal{H})=0$. We propose the following allocation rule.

Definition 6 The Myerson value for complete coalition structures is a mapping $f: \mathbf{C C S} \rightarrow \mathbb{R}^{N}$ given by $f(\mathcal{H})=\phi\left(v^{\mathcal{H}}\right)$ for each $\mathcal{H} \in \mathbf{C C S}$ where $v^{\mathcal{H}}$ is the $\mathcal{H}$-projected game of $v$.

This is an allocation rule on complete coalition structures because if $i \notin S$ for each $S \in \mathcal{H}$ then $i$ is a null player in $v^{\mathcal{H}}$ and thus $f_{i}(\mathcal{H})=\phi_{i}\left(v^{\mathcal{H}}\right)=0$. This allocation rule restricted to union stable structures coincides with the Myerson value for union stable structures studied by Algaba et al. (2001). To see this, for $\mathcal{H} \in$ USS, define

$$
C(\mathcal{H}) \equiv\{T \in \mathcal{H} \mid T \text { is maximal in } \mathcal{H}\}
$$

which is a partition of $\bigcup_{T \in \mathcal{H}} T$. For a game $v$ and a union stable structure $\mathcal{H}$, a game $r^{\mathcal{H}}$ is the $\mathcal{H}$-restricted game of $v$ if

$$
r^{\mathcal{H}}(S)=\sum_{T \in C\left(\mathcal{H}_{S}\right)} v(T) \text { for each } S \in 2^{N}
$$

Note that $\mathcal{H}_{S}$ is also a union stable structure. An allocation rule $f:$ USS $\rightarrow \mathbb{R}^{N}$ is the Myerson value for union stable structures if $f(\mathcal{H})=\phi\left(r^{\mathcal{H}}\right)$ for each $\mathcal{H} \in$ USS. Since $r^{\mathcal{H}}$ coincides with $v^{\mathcal{H}}$ as the next lemma shows, the restriction of the Myerson value for complete coalition structures to USS coincides with that for union stable structures.

Lemma 7 For a game $v$ and a union stable structure $\mathcal{H}$, the $\mathcal{H}$-restricted game of $v$ is the $\mathcal{H}$ projected game of $v$, i.e., $r^{\mathcal{H}}=v^{\mathcal{H}}$.

Proof. Since $C\left(\mathcal{H}_{S}\right) \subseteq \mathcal{H}_{S} \subseteq \mathcal{H}$ for $S \in 2^{N}, r^{\mathcal{H}}(S)=\sum_{T \in C\left(\mathcal{H}_{S}\right)} v(T)=\sum_{T \in C\left(\mathcal{H}_{S}\right)} v^{\mathcal{H}}(T)$ by Lemma 1. Since each $T \in C\left(\mathcal{H}_{S}\right)$ is a maximal element of $\mathcal{H}_{S}$, if $S^{\prime} \subseteq S$ and $S^{\prime} \nsubseteq T$ for each $T \in C\left(\mathcal{H}_{S}\right)$, then we must have $S^{\prime} \notin \mathcal{H}_{S}$ and thus $S^{\prime} \notin \mathcal{H}$. Since $\beta_{S^{\prime}}^{\mathcal{H}}=0$ for each $S^{\prime} \notin \mathcal{H}$, we have $v^{\mathcal{H}}(S)=\sum_{T \subseteq S} \beta_{T}^{\mathcal{H}}=\sum_{T \in C\left(\mathcal{H}_{S}\right)} \sum_{R \subseteq T} \beta_{R}^{\mathcal{H}}=\sum_{T \in C\left(\mathcal{H}_{S}\right)} v^{\mathcal{H}}(T)$. Therefore, $v^{\mathcal{H}}(S)=r^{\mathcal{H}}(S)$.

We characterize the Myerson value for complete coalition structures in terms of the following axioms for allocation rules on complete coalition structures. ${ }^{7}$

\footnotetext{
${ }^{7}$ Algaba et al. (2001) provide an axiomatization for the Myerson value for union stable structures. Their set of axioms is similar to that of the original Myerson value (Myerson, 1977), which consists of fairness and component efficiency. Thus, the set of axioms of Algaba et al. (2001) is different from ours.
} 
Feasible coalition efficiency (FE) For each $\mathcal{H} \in \mathbf{C C S}$, if $S \in \mathcal{H}$ and $\mathcal{H}_{S}=\mathcal{H}$, then

$$
\sum_{i \in S} f_{i}(\mathcal{H})=v(S)
$$

Balanced contribution (BC) For each $\mathcal{H} \in \mathbf{C C S}$ and $i, j \in N$,

$$
f_{i}(\mathcal{H})-f_{i}\left(\mathcal{H}_{-j}\right)=f_{j}(\mathcal{H})-f_{j}\left(\mathcal{H}_{-i}\right)
$$

No contribution by unassociated players (NC) For each $\mathcal{H} \in$ CCS, if $i, j \in N$ are not $\mathcal{H}$-associated in $N$, then

$$
f_{i}(\mathcal{H})-f_{i}\left(\mathcal{H}_{-j}\right)=0
$$

Fairness (FA) For each $\mathcal{H} \in \mathbf{C C S}$, if $i, j \in H \in \mathcal{H}$ and $\mathcal{H} \backslash\{H\} \in \mathbf{C C S}$, then

$$
f_{i}(\mathcal{H})-f_{i}(\mathcal{H} \backslash\{H\})=f_{j}(\mathcal{H})-f_{j}(\mathcal{H} \backslash\{H\}) .
$$

FE states that if $S$ is feasible and all players outside $S$ stand alone, then the members of $S$ allocate to themselves the total wealth $v(S)$ available to them. ${ }^{8}$ BC states that player $j$ 's contribution to $i$ equals $i$ 's contribution to $j$. NC states that player $j$ 's contribution to $i$ equals zero if $i$ and $j$ are not $\mathcal{H}$-associated in $N$. FA states that if a feasible coalition is removed and the resulting set remains to be a complete coalition structure, then the changes of payoffs are the same for all players in the feasible coalition. Myerson $(1977,1980)$ introduces FA and BC and shows that BC implies FA in his formulation. The reader may wonder if the presumption in FA in our formulation may be vacuous in some cases, but the next lemma shows that it is not the case.

Lemma 8 For $\mathcal{H} \in \mathbf{C C S}$, let $i, j \in N$ with $i \neq j$ be $\mathcal{H}$-associated in $N$. Then, there exists $H \in \mathcal{H}$ such that $i, j \in H$ and $\mathcal{H} \backslash\{H\} \in \mathbf{C C S}$.

Proof. By definition, $i, j \in N$ are $\mathcal{H}$-associated in $N$ if and only if the set $\{S \in \mathcal{H} \mid\{i, j\} \subseteq S\}$ is nonempty. Let $H \in\{S \in \mathcal{H} \mid\{i, j\} \subseteq S\}$ be a minimal element of the set in the set inclusion order; that is, there does not exist $S^{\prime} \in\{S \in \mathcal{H} \mid\{i, j\} \subseteq S\}$ with $S^{\prime} \subsetneq H$. We show that $\mathcal{H} \backslash\{H\} \in$ CCS. Seeking a contradiction, suppose that $\mathcal{H} \backslash\{H\} \notin$ CCS. Then, there exists a $\mathcal{H} \backslash\{H\}$-complete coalition $H^{\prime}$ with $H^{\prime} \notin \mathcal{H} \backslash\{H\}$. By the definition of $\mathcal{H}$-completeness, every $\mathcal{H} \backslash\{H\}$-complete coalition is $\mathcal{H}$-complete and thus $H^{\prime}$ is an element of $\mathcal{H}$ since $\mathcal{H} \in \mathbf{C C S}$. Thus, $H^{\prime} \in \mathcal{H} \backslash(\mathcal{H} \backslash\{H\})$, which implies that $H^{\prime}=H$. Therefore, $H$ is $\mathcal{H} \backslash\{H\}$-complete. On the other hand, since $H$ is a minimal element of $\{S \in \mathcal{H} \mid\{i, j\} \subseteq S\}$, there does not exist $S^{\prime} \in(\mathcal{H} \backslash\{H\})_{H}$ with $i, j \in S^{\prime}$, which implies that $H$ is not $\mathcal{H} \backslash\{H\}$-complete, a contradiction.

We are ready to state our main result, which characterizes the Myerson value for complete coalition structures in terms of FE, NC, and either FA or BC. We prove it in the next section.

Proposition 2 The following four statements about an allocation rule $f: \mathbf{C C S} \rightarrow \mathbb{R}^{N}$ on complete coalition structures are equivalent.

(i) $f$ is the Myerson value for complete coalition structures.

(ii) $f$ satisfies $F E, N C$, and $F A$.

\footnotetext{
${ }^{8}$ Amer and Carreras (1995) consider a different type of feasibility requirement.
} 
(iii) $f$ satisfies $F E, N C$, and $B C$.

(iv) $f(\mathcal{H})$ is the vector of the marginal contributions of a game $p^{\mathcal{H}}$ satisfying the following two conditions:

- If $S \in \mathcal{H}$, then

$$
\sum_{i \in S}\left(p^{\mathcal{H}}(S)-p^{\mathcal{H}}(S \backslash\{i\})\right)=v(S)
$$

- If $S \notin \mathcal{H}$ and $i, j \in S$ are not $\mathcal{H}$-associated in $S$, then

$$
p^{\mathcal{H}}(S)-p^{\mathcal{H}}(S \backslash\{i\})=p^{\mathcal{H}}(S \backslash\{j\})-p^{\mathcal{H}}(S \backslash\{i, j\}) .
$$

That is, $f_{i}(\mathcal{H})=p^{\mathcal{H}}(N)-p^{\mathcal{H}}(N \backslash\{i\})$ for each $i \in N$ and $\mathcal{H} \in \mathbf{C C S}$.

Notice the resemblance between $p^{\mathcal{H}}$ in (iv) and the potential for $v$. The latter satisfies (3) for each $S \in 2^{N}$, whereas the former satisfies (3) for each $S \in \mathcal{H}$, which is condition (5). Condition (6) requires that the marginal contribution of player $i$ to $p^{\mathcal{H}}(S)$ be determined by players who are $\mathcal{H}$-associated with $i$ in $S$. Note that if $\mathcal{H}=2^{N}$, then (5) is identical to (3), and (6) holds trivially because any pair of players are $\mathcal{H}$-associated in any coalition containing them. Thus in this case, $p^{\mathcal{H}}$ coincides with the potential for $v$ by Proposition 1. As will be shown in Lemma 15 in the next section, $p^{\mathcal{H}}$ turns out to be the potential for $v^{\mathcal{H}}$, which will explain why the allocation rule is uniquely determined.

The following numerical examples illustrate the difference between the Myerson value for complete coalition structures and that for union stable structures.

Example 1 For $n \geq 4$, let $S_{1}, S_{2} \subsetneq N$ be such that $\left|S_{1}\right| \geq 2,\left|S_{2}\right| \geq 2, S_{1} \cup S_{2}=\{2, \ldots, n\}$ and $S_{1} \cap S_{2}=\{n\}$. Consider $\mathcal{H}^{1}=\left\{\{1\}, S_{1}, S_{2}, N\right\}$ and $\mathcal{H}^{2}=\left\{\{1\}, S_{1}, S_{2}, S_{1} \cup S_{2}, N\right\}$. Note that $\mathcal{H}^{1}, \mathcal{H}^{2} \in \mathbf{C C S}, \mathcal{H}^{1} \notin \mathbf{U S S}$, and $\mathcal{H}^{2} \in \mathbf{U S S}$. Define a game $v$ by

$$
v=u_{\{1\}}+u_{\{2, \ldots, n\}}+u_{N} .
$$

Then, we have $v^{\mathcal{H}^{1}}=u_{\{1\}}+2 u_{N}$ and $v^{\mathcal{H}^{2}}=v$. Thus, by (2), the payoff vectors of the Myerson value for complete coalition structures are as follows.

\begin{tabular}{c|cc} 
& player 1 & player $i \in\{2, \ldots, n\}$ \\
\hline$f\left(\mathcal{H}^{1}\right)$ & $1+2 / \mathrm{n}$ & $2 / \mathrm{n}$ \\
$f\left(\mathcal{H}^{2}\right)$ & $1+1 / \mathrm{n}$ & $1 /(\mathrm{n}-1)+1 / \mathrm{n}$
\end{tabular}

Since $\mathcal{H}^{1} \notin$ USS, the Myerson value for union stable structures does not determine payoff vectors for $\mathcal{H}^{1}$, and since $\mathcal{H}^{2}$ is the minimal union stable structure containing $\mathcal{H}^{1}$, the Myerson value in the sense of Myerson (1980) and van den Nouweland et al. (1992) assigns the same payoff vector $f\left(\mathcal{H}^{2}\right)=\phi\left(r^{\mathcal{H}^{2}}\right)$ to $\mathcal{H}^{1}$. The Myerson value for complete coalition structures provides one possible way to assign different payoff vectors to $\mathcal{H}^{1}$ and $\mathcal{H}^{2}$. Observe that $f_{1}\left(\mathcal{H}^{1}\right)>f_{1}\left(\mathcal{H}^{2}\right)$ and $f_{i}\left(\mathcal{H}^{1}\right)<f_{i}\left(\mathcal{H}^{2}\right)$ for $i \in S_{1} \cup S_{2}=\{2, \ldots, n\}$. This can be interpreted as follows. An unfeasible coalition $S_{1} \cup S_{2}$ in $\mathcal{H}^{1}$ is feasible in $\mathcal{H}^{2}$, and the bargaining power of $S_{1} \cup S_{2}$ is larger in $\mathcal{H}^{2}$ than in $\mathcal{H}^{1}$, resulting in a larger payoff to player $i \in S_{1} \cup S_{2}$ and a smaller payoff to player 1 . 
Example 2 For $n=5$, consider

$$
\begin{aligned}
& \mathcal{H}^{1}=\{\{1\},\{2,3\},\{3,4\},\{4,5\}, N\}, \\
& \mathcal{H}^{2}=\{\{1\},\{2,3\},\{3,4\},\{4,5\},\{2,3,4\},\{3,4,5\}, N\}, \\
& \mathcal{H}^{3}=\{\{1\},\{2,3\},\{3,4\},\{4,5\},\{2,3,4\},\{3,4,5\},\{2,3,4,5\}, N\} .
\end{aligned}
$$

Note that $\mathcal{H}^{1}, \mathcal{H}^{2}, \mathcal{H}^{3} \in \mathbf{C C S}, \mathcal{H}^{1}, \mathcal{H}^{2} \notin \mathbf{U S S}$, and $\mathcal{H}^{3} \in \mathbf{U S S}$. Define a game $v$ by

$$
v=u_{\{1\}}+u_{\{2,3,4\}}+u_{\{3,4,5\}}+u_{\{2,3,4,5\}}+u_{N} .
$$

Then, we have $v^{\mathcal{H}^{1}}=u_{\{1\}}+4 u_{N}, v^{\mathcal{H}^{2}}=u_{\{1\}}+u_{\{2,3,4\}}+u_{\{3,4,5\}}+2 u_{N}$, and $v^{\mathcal{H}^{3}}=v$. Thus, by (2), the payoff vectors of the Myerson value for complete coalition structures are as follows.

\begin{tabular}{c|ccc} 
& player 1 & player $i \in\{2,5\}$ & player $i \in\{3,4\}$ \\
\hline$f\left(\mathcal{H}^{1}\right)$ & $9 / 5$ & $4 / 5$ & $4 / 5$ \\
$f\left(\mathcal{H}^{2}\right)$ & $7 / 5$ & $11 / 15$ & $16 / 15$ \\
$f\left(\mathcal{H}^{3}\right)$ & $6 / 5$ & $47 / 60$ & $67 / 60$
\end{tabular}

Observe that $f_{1}\left(\mathcal{H}^{1}\right)>f_{1}\left(\mathcal{H}^{2}\right)>f_{1}\left(\mathcal{H}^{3}\right)$ and $\sum_{i \neq 1} f_{i}\left(\mathcal{H}^{1}\right)<\sum_{i \neq 1} f_{i}\left(\mathcal{H}^{2}\right)<\sum_{i \neq 1} f_{i}\left(\mathcal{H}^{3}\right)$. When more coalitions to which player 1 does not belong are feasible, player 1 has smaller bargaining power, which results in a smaller payoff to player 1 .

When we apply the Myerson value for complete coalition structures for a given partial cooperation structure, it is important to carefully identify a set of feasible coalitions $\mathcal{H}$ describing the partial cooperation structure. To address this issue, let $\overline{\mathcal{H}}$ denote the minimal union stable structure containing $\mathcal{H} \in \mathbf{C C S},{ }^{9}$ and assume that we require $\sum_{i \in S} f_{i}(\mathcal{H})=v(S)$ for each $S \in C(\overline{\mathcal{H}}),{ }^{10}$ which is not always true in the Myerson value for complete coalition structures. This requirement implies that, for each $S \in C(\overline{\mathcal{H}})$, the total wealth $v(S)$ is available to the members of $S$; that is, each $S \in C(\overline{\mathcal{H}})$ is feasible. Thus, when we require $\sum_{i \in S} f_{i}(\mathcal{H})=v(S)$ for each $S \in C(\overline{\mathcal{H}})$, we must choose $\mathcal{H}$ satisfying $C(\overline{\mathcal{H}}) \subseteq \mathcal{H}$ to be logically consistent in the analysis. If this is the case, we indeed have $\sum_{i \in S} f_{i}(\mathcal{H})=v(S)$ for each $S \in C(\overline{\mathcal{H}})$ as the following lemma shows.

Lemma 9 Let $f: \mathbf{C C S} \rightarrow \mathbb{R}^{N}$ be the Myerson value for complete coalition structures. If $\mathcal{H} \in$ CCS and $C(\overline{\mathcal{H}}) \subseteq \mathcal{H}$, then $\sum_{i \in S} f_{i}(\mathcal{H})=v(S)$ for each $S \in C(\overline{\mathcal{H}})$.

Proof. Suppose that $C(\overline{\mathcal{H}}) \subseteq \mathcal{H}$. Then, for each $S \in C(\overline{\mathcal{H}})$, it holds that $S \in \mathcal{H}_{S}=\left(\mathcal{H}_{S}\right)_{S}$. Thus, $\sum_{i \in S} f_{i}\left(\mathcal{H}_{S}\right)=v(S)$ by FE. Since $i \in S$ and $j \notin S$ are not associated in $N, f_{i}\left(\mathcal{H}_{S}\right)=f_{i}(\mathcal{H})$ by $\mathrm{NC}$, and thus $\sum_{i \in S} f_{i}(\mathcal{H})=v(S)$ for each $S \in C(\overline{\mathcal{H}})$.

\section{The proof}

This section provides the proof of Proposition 2. It proceeds in the following order: (i) $\Rightarrow$ (ii) $\Rightarrow$ (iii) $\Rightarrow$ (iv) $\Leftrightarrow($ i).

\footnotetext{
${ }^{9}$ As shown by Algaba et al. (2000), $\overline{\mathcal{H}}$ is constructed by the following procedure: by setting $\mathcal{H}^{(0)}=\mathcal{H}, \mathcal{H}^{(k)}=$ $\left\{S \cup T \mid S, T \in \mathcal{H}^{(k-1)}, S \cap T \neq \emptyset\right\}$ for $k \geq 1$, we obtain $\overline{\mathcal{H}}=\mathcal{H}^{(k)}$ where $k$ is the smallest integer with $\mathcal{H}^{(k)}=$ $\mathcal{H}^{(k+1)}$.

${ }^{10}$ This requirement is called component efficiency, and the Myerson value for union stable structures satisfies it (Myerson, 1977; Algaba et al., 2001).
} 


\section{$5.1 \quad$ (i) $\Rightarrow($ ii $)$}

The following lemma plays an important role.

Lemma 10 Let $v^{\mathcal{H}}=\sum_{T \in 2^{N}} \beta_{T}^{\mathcal{H}} u_{T}$ and $v^{\mathcal{H}_{S}}=\sum_{T \in 2^{N}} \beta_{T}^{\mathcal{H}_{S}} u_{T}$ for $\mathcal{H} \subseteq 2^{N}$ and $S \in 2^{N}$. Then, $\beta_{T}^{\mathcal{H}}=\beta_{T}^{\mathcal{H}_{S}}$ for each $T \subseteq S$.

Proof. By the construction of $\beta_{T}^{\mathcal{H}},\left\{\beta_{T}^{\mathcal{H}}\right\}_{T \subseteq S}$ is determined by $\{v(R)\}_{R \in \mathcal{H}_{S}}$, and so is $\left\{\beta_{T}^{\mathcal{H}_{S}}\right\}_{T \subseteq S}$. Furthermore, the recursive procedure is the same for both $\left\{\beta_{T}^{\mathcal{H}}\right\}_{T \subseteq S}$ and $\left\{\beta_{T}^{\mathcal{H}_{S}}\right\}_{T \subseteq S}$. Therefore, $\beta_{T}^{\mathcal{H}}=\beta_{T}^{\mathcal{H}_{S}}$ for each $T \subseteq S$.

We are ready to establish (i) $\Rightarrow$ (ii).

Lemma 11 Let $f: \mathbf{C C S} \rightarrow \mathbb{R}^{N}$ be the Myerson value for complete coalition structures. Then, $f$ satisfies $F E, N C$, and $F A$.

Proof. Let $v^{\mathcal{H}}=\sum_{T \in 2^{N}} \beta_{T}^{\mathcal{H}} u_{T}$ be the $\mathcal{H}$-projected game of $v$. For $S \in \mathcal{H} \in \mathbf{C C S}$, suppose that $\mathcal{H}_{S}=\mathcal{H}$. Then, by the definition of $v^{\mathcal{H}}, \beta_{T}^{\mathcal{H}}=0$ for each $T \nsubseteq S$. Thus,

$$
f_{i}(\mathcal{H})=\phi_{i}\left(v^{\mathcal{H}}\right)=\sum_{T \in 2^{N}: i \in T} \beta_{T}^{\mathcal{H}} /|T|=\sum_{T \in 2^{S}: i \in T} \beta_{T}^{\mathcal{H}} /|T|
$$

Accordingly,

$$
\sum_{i \in S} f_{i}(\mathcal{H})=\sum_{i \in S} \sum_{T \in 2^{S}: i \in T} \beta_{T}^{\mathcal{H}} /|T|=\sum_{T \in 2^{S}} \beta_{T}^{\mathcal{H}}=v^{\mathcal{H}}(S)=v(S)
$$

since $S \in \mathcal{H}$, which implies FE.

For $\mathcal{H} \in \mathbf{C C S}$, suppose that $i, j \in N$ are not $\mathcal{H}$-associated in $N$. Then, for $T \subseteq N$ with $i, j \in T$, it holds that $T \notin \mathcal{H}$ and $T \notin \mathcal{H}_{-j}$, which implies $\beta_{T}^{\mathcal{H}}=\beta_{T}^{\mathcal{H}_{-j}}=0$. On the other hand, by Lemma $10, \beta_{T}^{\mathcal{H}}=\beta_{T}^{\mathcal{H}_{-j}}$ for each $T \subseteq N \backslash\{j\}$. Therefore, $\beta_{T}^{\mathcal{H}}=\beta_{T}^{\mathcal{H}_{-j}}$ whenever $i \in T$, and thus

$$
f_{i}(\mathcal{H})=\phi_{i}\left(v^{\mathcal{H}}\right)=\sum_{T \in 2^{N}: i \in T} \beta_{T}^{\mathcal{H}} /|T|=\sum_{T \in 2^{N}: i \in T} \beta_{T}^{\mathcal{H}_{-j}} /|T|=\phi_{i}\left(v^{\mathcal{H}_{-j}}\right)=f_{i}\left(\mathcal{H}_{-j}\right),
$$

which implies NC.

For $\mathcal{H} \in \mathbf{C C S}$, suppose that $i, j \in H \in \mathcal{H}$ and $\mathcal{H} \backslash\{H\} \in \mathbf{C C S}$. Then,

$$
\begin{aligned}
f_{i}(\mathcal{H})-f_{j}(\mathcal{H}) & =\sum_{T \in 2^{N}: i \in T} \beta_{T}^{\mathcal{H}} /|T|-\sum_{T \in 2^{N}: j \in T} \beta_{T}^{\mathcal{H}} /|T| \\
& =\sum_{T \in 2^{N \backslash\{j\}: i \in T}} \beta_{T}^{\mathcal{H}} /|T|-\sum_{T \in 2^{N \backslash\{i\}: j \in T}} \beta_{T}^{\mathcal{H}} /|T|, \\
f_{i}(\mathcal{H} \backslash\{H\})-f_{j}(\mathcal{H} \backslash\{H\}) & =\sum_{T \in 2^{N}: i \in T} \beta_{T}^{\mathcal{H} \backslash\{H\}} /|T|-\sum_{T \in 2^{N}: j \in T} \beta_{T}^{\mathcal{H} \backslash\{H\}} /|T| \\
& =\sum_{T \in 2^{N \backslash\{j\}: i \in T}} \beta_{T}^{\mathcal{H} \backslash\{H\}} /|T|-\sum_{T \in 2^{N \backslash\{i\}: j \in T}} \beta_{T}^{\mathcal{H} \backslash\{H\}} /|T| .
\end{aligned}
$$

Since $\mathcal{H}_{-j}=(\mathcal{H} \backslash\{H\})_{-j}$, if $T \subseteq N \backslash\{j\}$ then $\beta_{T}^{\mathcal{H} \backslash\{H\}}=\beta_{T}^{(\mathcal{H} \backslash\{H\})_{-j}}=\beta_{T}^{\mathcal{H}_{-j}}=\beta_{T}^{\mathcal{H}}$ by Lemma 10. Similarly, if $T \subseteq N \backslash\{i\}$ then $\beta_{T}^{\mathcal{H} \backslash\{H\}}=\beta_{T}^{\mathcal{H}}$. Therefore, by (7) and (8), we have

$$
f_{i}(\mathcal{H})-f_{j}(\mathcal{H})=f_{i}(\mathcal{H} \backslash\{H\})-f_{j}(\mathcal{H} \backslash\{H\}),
$$

which implies FA. 


\section{$5.2 \quad$ (ii) $\Rightarrow$ (iii)}

As the next result shows, FA and NC together imply BC. Thus, if an allocation rule satisfies FE, $\mathrm{NC}$, and FA, then it satisfies FE, NC, and $\mathrm{BC}$, establishing (ii) $\Rightarrow$ (iii).

Lemma 12 If an allocation rule $f: \mathbf{C C S} \rightarrow \mathbb{R}^{N}$ satisfies $F A$ and $N C$, then it satisfies $B C$.

Proof. Suppose that $i, j \in N$ with $i \neq j$ are not $\mathcal{H}$-associated in $N$. Then, NC implies that $f_{i}(\mathcal{H})-f_{i}\left(\mathcal{H}_{-j}\right)=f_{j}(\mathcal{H})-f_{j}\left(\mathcal{H}_{-i}\right)=0$.

Suppose that $i, j \in N$ with $i \neq j$ are $\mathcal{H}$-associated in $N$, which is true if and only if $\{S \in$ $\mathcal{H} \mid\{i, j\} \subseteq S\} \neq \emptyset$. We first show that

$$
f_{i}(\mathcal{H})-f_{i}(\mathcal{H} \backslash\{S \in \mathcal{H} \mid\{i, j\} \subseteq S\})=f_{j}(\mathcal{H})-f_{j}(\mathcal{H} \backslash\{S \in \mathcal{H} \mid\{i, j\} \subseteq S\})
$$

By Lemma 8, there exists $H_{1} \in \mathcal{H}$ with $i, j \in H_{1}$ and $\mathcal{H} \backslash\left\{H_{1}\right\} \in$ CCS. By FA, $f_{i}(\mathcal{H})-$ $f_{i}\left(\mathcal{H} \backslash\left\{H_{1}\right\}\right)=f_{j}(\mathcal{H})-f_{j}\left(\mathcal{H} \backslash\left\{H_{1}\right\}\right)$. This is $(9)$ if $\{S \in \mathcal{H} \mid\{i, j\} \subseteq S\}=\left\{H_{1}\right\}$. Otherwise, $i$ and $j$ are $\mathcal{H} \backslash\left\{H_{1}\right\}$-associated in $N$. Then, by the same argument, there exists $H_{2} \in \mathcal{H}$ with $i, j \in H_{2}$, $\mathcal{H} \backslash\left\{H_{1}, H_{2}\right\} \in \mathbf{C C S}$, and $f_{i}\left(\mathcal{H} \backslash\left\{H_{1}\right\}\right)-f_{i}\left(\mathcal{H} \backslash\left\{H_{1}, H_{2}\right\}\right)=f_{j}\left(\mathcal{H} \backslash\left\{H_{1}\right\}\right)-f_{j}\left(\mathcal{H} \backslash\left\{H_{1}, H_{2}\right\}\right)$, which implies that $f_{i}(\mathcal{H})-f_{i}\left(\mathcal{H} \backslash\left\{H_{1}, H_{2}\right\}\right)=f_{j}(\mathcal{H})-f_{j}\left(\mathcal{H} \backslash\left\{H_{1}, H_{2}\right\}\right)$. This is (9) if $\{S \in \mathcal{H} \mid\{i, j\} \subseteq$ $S\}=\left\{H_{1}, H_{2}\right\}$. Otherwise, $i$ and $j$ are $\mathcal{H} \backslash\left\{H_{1}, H_{2}\right\}$-associated in $N$. Repeating this, we obtain (9) when $\{S \in \mathcal{H} \mid\{i, j\} \subseteq S\}=\left\{H_{1}, \ldots, H_{k}\right\}$. Note that $i$ and $j$ are not $\mathcal{H} \backslash\left\{H_{1}, \ldots, H_{k}\right\}$-associated in $N$ since $\mathcal{H} \backslash\left\{H_{1}, \ldots, H_{k}\right\}=\{S \in \mathcal{H} \mid\{i, j\} \nsubseteq S\}$. Thus, NC implies that

$$
f_{i}\left(\mathcal{H} \backslash\left\{H_{1}, \ldots, H_{k}\right\}\right)=f_{i}\left(\left(\mathcal{H} \backslash\left\{H_{1}, \ldots, H_{k}\right\}\right)_{-j}\right)=f_{i}\left(\mathcal{H}_{-j}\right),
$$

where the latter equality holds because $\left(\mathcal{H} \backslash\left\{H_{1}, \ldots, H_{k}\right\}\right)_{-j}=\{S \in \mathcal{H} \mid\{i, j\} \nsubseteq S$ and $j \notin S\}=$ $\{S \in \mathcal{H} \mid j \notin S\}=\mathcal{H}_{-j}$. Similarly, it follows that $f_{j}\left(\mathcal{H} \backslash\left\{H_{1}, \ldots, H_{k}\right\}\right)=f_{j}\left(\mathcal{H}_{-i}\right)$. By plugging this and (10) into (9), we have established BC.

\section{$5.3 \quad$ (iii) $\Rightarrow$ (iv)}

As noted by Hart and Mas-Colell (1989), BC is a finite difference analogue of the Frobenius integrability condition, i.e., the symmetry of the cross partial derivatives, which suggests that the solution admits a potential. In fact, BC assures the existence of a "potential" in the following sense. ${ }^{11}$

Lemma 13 If an allocation rule $f: \mathbf{C C S} \rightarrow \mathbb{R}^{N}$ satisfies $B C$, then, for each $\mathcal{H} \in \mathbf{C C S}$, there exists a game $p^{\mathcal{H}}$ such that $f_{i}\left(\mathcal{H}_{S}\right)=p^{\mathcal{H}}(S)-p^{\mathcal{H}}(S \backslash\{i\})$ for each $i \in S$ and $S \in 2^{N}$.

Proof. Note that $\mathcal{H}_{S} \in \mathbf{C C S}$ by Lemma 3. Define a game $p^{\mathcal{H}}$ by the following rule: for each $S=\left\{i_{1}, \ldots, i_{k}\right\} \in 2^{N}$ with $i_{1}<\cdots<i_{k}, p^{\mathcal{H}}(S)=\sum_{l=1}^{k} f_{i_{l}}\left(\mathcal{H}_{\left\{i_{1}, \ldots, i_{l}\right\}}\right)$. By construction, if $i=\max S$, then $f_{i}\left(\mathcal{H}_{S}\right)=p^{\mathcal{H}}(S)-p^{\mathcal{H}}(S \backslash\{i\})$.

We show by induction that $f_{i}\left(\mathcal{H}_{S}\right)=p^{\mathcal{H}}(S)-p^{\mathcal{H}}(S \backslash\{i\})$ for each $i \in S$ and $S \in 2^{N}$. If $|S|=1$ and $S=\{i\}$, then $f_{i}\left(\mathcal{H}_{\{i\}}\right)=p^{\mathcal{H}}(\{i\})-p^{\mathcal{H}}(\emptyset)$. Suppose as an induction hypothesis that $f_{i}\left(\mathcal{H}_{S}\right)=$

\footnotetext{
${ }^{11}$ Consider a vector-valued mapping $F: \mathbb{R}^{n} \rightarrow \mathbb{R}^{n}$. In vector analysis, a function $f: \mathbb{R}^{n} \rightarrow \mathbb{R}$ is said to be a potential of $F$ if $F=\left(\partial f / \partial x_{i}\right)_{i=1}^{n}$.
} 
$p^{\mathcal{H}}(S)-p^{\mathcal{H}}(S \backslash\{i\})$ for each $i \in S$ and $S \in 2^{N}$ with $|S| \leq k<n$. Let $S=\left\{i_{1}, \ldots, i_{k+1}\right\} \in 2^{N}$ with $i_{1}<\cdots<i_{k+1}$. For every $i \in S$, by applying BC (with $\mathcal{H}_{S}$ instead of $\mathcal{H}$ ), we have

$$
\begin{aligned}
f_{i}\left(\mathcal{H}_{S}\right) & =f_{i_{k+1}}\left(\mathcal{H}_{S}\right)-f_{i_{k+1}}\left(\left(\mathcal{H}_{S}\right)_{-i}\right)+f_{i}\left(\left(\mathcal{H}_{S}\right)_{-i_{k+1}}\right) \\
& =f_{i_{k+1}}\left(\mathcal{H}_{S}\right)-f_{i_{k+1}}\left(\mathcal{H}_{S \backslash\{i\}}\right)+f_{i}\left(\mathcal{H}_{S \backslash\left\{i_{k+1}\right\}}\right) .
\end{aligned}
$$

By the construction of $p^{\mathcal{H}}$,

$$
f_{i_{k+1}}\left(\mathcal{H}_{S}\right)=p^{\mathcal{H}}(S)-p^{\mathcal{H}}\left(S \backslash\left\{i_{k+1}\right\}\right) .
$$

By the induction hypothesis,

$$
\begin{aligned}
& f_{i_{k+1}}\left(\mathcal{H}_{S \backslash\{i\}}\right)=p^{\mathcal{H}}(S \backslash\{i\})-p^{\mathcal{H}}\left(S \backslash\left\{i, i_{k+1}\right\}\right), \\
& f_{i}\left(\mathcal{H}_{S \backslash\left\{i_{k+1}\right\}}\right)=p^{\mathcal{H}}\left(S \backslash\left\{i_{k+1}\right\}\right)-p^{\mathcal{H}}\left(S \backslash\left\{i, i_{k+1}\right\}\right) .
\end{aligned}
$$

Plugging (12), (13), and (14) into (11), we have $f_{i}\left(\mathcal{H}_{S}\right)=p^{\mathcal{H}}(S)-p^{\mathcal{H}}(S \backslash\{i\})$.

A "potential" in Lemma 13 is shown to satisfy (5) and (6) if an allocation rule satisfies FE and $\mathrm{NC}$ in addition.

Lemma 14 Let an allocation rule $f: \mathbf{C C S} \rightarrow \mathbb{R}^{N}$ satisfy $F E$ and $N C$. For $\mathcal{H} \in \mathbf{C C S}$, suppose that there exists a game $p^{\mathcal{H}}$ such that $f_{i}\left(\mathcal{H}_{S}\right)=p^{\mathcal{H}}(S)-p^{\mathcal{H}}(S \backslash\{i\})$ for each $i \in S$ and $S \in 2^{N}$. Then, $p^{\mathcal{H}}$ satisfies (5) and (6).

Proof. For $\mathcal{H} \in \mathbf{C C S}$, suppose that $S \in \mathcal{H}$. By FE, $\sum_{i \in S} f_{i}\left(\mathcal{H}_{S}\right)=\sum_{i \in S}\left(p^{\mathcal{H}}(S)-p^{\mathcal{H}}(S \backslash\{i\})\right)=$ $v(S)$. Therefore, $p^{\mathcal{H}}$ satisfies (5).

For $\mathcal{H} \in \mathbf{C C S}$, suppose that $S \notin \mathcal{H}$ and that $i, j \in S$ are not $\mathcal{H}$-associated in $S$. It is clear that $i$ and $j$ are not $\mathcal{H}_{S}$-associated in $N$. Thus, by NC, $p^{\mathcal{H}}(S)-p^{\mathcal{H}}(S \backslash\{i\})=f_{i}\left(\mathcal{H}_{S}\right)=f_{i}\left(\left(\mathcal{H}_{S}\right)_{-j}\right)=$ $f_{i}\left(\mathcal{H}_{S \backslash\{j\}}\right)=p^{\mathcal{H}}(S \backslash\{j\})-p^{\mathcal{H}}(S \backslash\{i, j\})$. Therefore, $p^{\mathcal{H}}$ satisfies (6).

By Lemma 13 and Lemma 14, if an allocation rule $f: \mathbf{C C S} \rightarrow \mathbb{R}$ satisfies FE, NC, and BC, then there exists a game $p^{\mathcal{H}}$ satisfying (5) and (6) such that $f_{i}(\mathcal{H})=f_{i}\left(\mathcal{H}_{N}\right)=p^{\mathcal{H}}(N)-p^{\mathcal{H}}(N \backslash\{i\})$, which establishes (iii) $\Rightarrow$ (iv).

\section{4 (iv) $\Leftrightarrow(\mathrm{i})$}

We shall show below that a game $p^{\mathcal{H}}$ which satisfies the conditions in (iv) must be the potential for $v^{\mathcal{H}}$. This suffices to establish (iv) $\Leftrightarrow$ (i) by Proposition 1 .

Lemma 15 Suppose that $\mathcal{H} \in \mathbf{C C S}$. Then, there exists a unique game $p^{\mathcal{H}}$ satisfying (5) and (6). The game $p^{\mathcal{H}}$ coincides with the potential for $v^{\mathcal{H}}$.

Proof. We first show that the potential for $v^{\mathcal{H}}$ does satisfy (5) and (6). Let $p^{\mathcal{H}}$ be the potential for $v^{\mathcal{H}}=\sum_{T \in 2^{N}} \beta_{T}^{\mathcal{H}} u_{T}$. Then by Proposition $1, p^{\mathcal{H}}=\sum_{T \in 2^{N}}\left(\beta_{T}^{\mathcal{H}} /|T|\right) u_{T}$. Observe that $\sum_{i \in S}\left(p^{\mathcal{H}}(S)-p^{\mathcal{H}}(S \backslash\{i\})\right)=v^{\mathcal{H}}(S)=v(S)$ if $S \in \mathcal{H}$, where the first equality holds because $p^{\mathcal{H}}$ is the potential for $v^{\mathcal{H}}$, and the second equality holds by Lemma 1. This is condition (5). Next, observe that, since $\beta_{T}^{\mathcal{H}}=0$ for each $T \notin \mathcal{H}$,

$$
\begin{aligned}
p^{\mathcal{H}}(S)-p^{\mathcal{H}}(S \backslash\{i\}) & =\sum_{T \in \mathcal{H}_{S}} \beta_{T}^{\mathcal{H}} /|T|-\sum_{T \in \mathcal{H}_{S \backslash\{i\}}} \beta_{T}^{\mathcal{H}} /|T| \\
& =\sum_{T \in \mathcal{H}_{S}: i \in T} \beta_{T}^{\mathcal{H}} /|T|,
\end{aligned}
$$


and similarly,

$$
p^{\mathcal{H}}(S \backslash\{j\})-p^{\mathcal{H}}(S \backslash\{i, j\})=\sum_{T \in \mathcal{H}_{S \backslash\{j\}}: i \in T} \beta_{T}^{\mathcal{H}} /|T|
$$

for $i, j \in S$. Now suppose that $S \notin \mathcal{H}$ and $i, j \in S$ are not $\mathcal{H}$-associated in $S$. Then, there is no $T \in \mathcal{H}_{S}$ such that $i, j \in T$. This implies that $\left\{T \in \mathcal{H}_{S} \mid i \in T\right\}=\left\{T \in \mathcal{H}_{S \backslash\{j\}} \mid i \in T\right\}$ and thus $p^{\mathcal{H}}(S)-p^{\mathcal{H}}(S \backslash\{i\})=p^{\mathcal{H}}(S \backslash\{j\})-p^{\mathcal{H}}(S \backslash\{i, j\})$ by (15) and (16). This is condition (6).

To complete the proof, we show that a game $p^{\mathcal{H}}$ satisfying (5) and (6) is unique, by constructing $p^{\mathcal{H}}$ recursively such that in the $k$-th step we determine the unique value of $p^{\mathcal{H}}(S)$ with $|S|=k$ from $p^{\mathcal{H}}\left(S^{\prime}\right)$ with $\left|S^{\prime}\right| \leq k-1$. Start with $p^{\mathcal{H}}(\emptyset)=0$ since $p^{\mathcal{H}}$ is a game. Consider the $k$-th step with $k \geq 1$ and pick any $S$ with $|S|=k$. Suppose that $S \in \mathcal{H}$. Then, (5) is rewritten as

$$
p^{\mathcal{H}}(S)=|S|^{-1}\left(v(S)+\sum_{i \in S} p^{\mathcal{H}}(S \backslash\{i\})\right) .
$$

Since $p^{\mathcal{H}}(S \backslash\{i\})$ on the right hand side is uniquely calculated for each $i \in N$ in the previous step, so is $p^{\mathcal{H}}(S)$ on the left hand side. Suppose that $S \notin \mathcal{H}$. Then, there exist $i, j \in S$ that are not $\mathcal{H}$-associated in $S$ since $\mathcal{H} \in$ CCS. So, by (6),

$$
p^{\mathcal{H}}(S)=p^{\mathcal{H}}(S \backslash\{i\})+p^{\mathcal{H}}(S \backslash\{j\})-p^{\mathcal{H}}(S \backslash\{i, j\}) .
$$

Since the terms on the right hand side are uniquely calculated in the earlier steps, so is $p^{\mathcal{H}}(S)$ on the left hand side. Note that $p^{\mathcal{H}}(S)$ in (17) does not depend upon the choice of $i$ and $j$ because (17) holds for any $i, j \in S$ that are not $\mathcal{H}$-associated in $S$. By the procedure above, we can uniquely determine $p^{\mathcal{H}}$ recursively, which establishes the uniqueness.

\section{Concluding remarks}

This paper has introduced complete coalition structures as sets of feasible coalitions and proposed the Myerson value for complete coalition structures as an allocation rule. It shows that an allocation rule is the Myerson value for complete coalition structures if and only if it satisfies FE, NC, and FA.

As concluding remarks, we point out other possible applications of complete coalition structures. The position value (Meesen, 1988; Borm et al., 1992) and the Hamiache value (Hamiache, 1999) are allocation rules on networks. Algaba et al. (2000) and Bilbao and López (2006) study the extensions of the position value and the Hamiache value to union stable structures, respectively. ${ }^{12}$ Therefore, it would be interesting to study the extensions of these allocation rules to complete coalition structures.

\section{References}

Algaba, E., Bilbao, J. M., Borm, P., López, J. J., 2000. The position value for union stable structures. Math. Meth. Oper. Res. 52, 221-236.

Algaba, E., Bilbao, J. M., Borm, P., López, J. J., 2001. The Myerson value for union stable structures. Math. Meth. Oper. Res. 54, 359-371.

\footnotetext{
${ }^{12}$ See also van den Nouweland et al. (1992).
} 
Algaba, E., Bilbao, J. M., van den Brink, R., Jiménez-Losada, A., 2004. Cooperative games on antimatroids. Discrete Math. 1-15.

Amer, R., Carreras, F., 1995. Games and cooperative indices. Int. J. Game Theory 24, 239-258.

Bilbao, J. M., 2000. Cooperative Games on Combinatorial Structures. Kluwer Academic Publishers, Boston.

Bilbao, J. M., Jiménez, N., López, J. J., 2006. A note on a value with incomplete communication. Games Econ. Behav. 54, 419-429.

Borm, P., Owen, G., Tijs, S., 1992. On the position value for communication situations. SIAM J. Discrete Math. 5, 305-320.

Faigle, U, Kern, W., 1992. The Shapley value for cooperative games under precedence constraints. Int. J. Game Theory 21, 249-266.

Gilles, R. P. Owen, G., 1999. Cooperative games and disjunctive permission structures. Working paper. Virginia Tech.

Gilles, R. P., Owen, G., van den Brink, R., 1992. Games with permission structures: the conjunctive approach. Int. J. Game Theory 20, 277-293.

Hamiache, G., 1999. A value with incomplete communication. Games Econ. Behav. 26, 59-78.

Hart, S., Mas-Colell, A., 1989. Potential, value, and consistency. Econometrica 57, 589-614.

Kajii, A., Kojima, H., Ui, T., 2007. Cominimum additive operators. J. Math. Econ. 43, 218-230.

Kajii, A., Kojima, H., Ui, T., 2008. Coextrema additive operators. In: Neogy, S. K., Das, A. K., Bapat, B. (Eds.), Modeling, Computation and Optimization. World Scientific, Singapore, pp. 73-95.

Meesen, R., 1988. Communication games. Master's Thesis (in Dutch). University of Nijmegen, The Netherlands.

Myerson, R. B., 1977. Graphs and cooperation in games. Math. Oper. Res. 2, 225-229.

Myerson, R. B., 1980. Conference structures and fair allocation rules. Int. J. Game Theory 9, 169-182.

Shapley, L., 1953. A value for $n$-person games. In: Kuhn, H., Tucker, A. (Eds.), Contributions to the Theory of Games II. Princeton Univ. Press, Princeton, pp. 307-317.

van den Nouweland, A., Borm. P., Tijs, S., 1992. Allocation rules for hypergraph communication situations. Int. J. Game Theory 20, 255-268. 